\title{
Cluster growth in far-from-equilibrium particle models with diffusion, detachment, reattachment and deposition
}

\author{
F. D. A. Aarão Reis ${ }^{1, *}$ and R. B. Stinchcombe ${ }^{2, \dagger}$ \\ ${ }^{1}$ Instituto de Física, Universidade Federal Fluminense, Avenida Litorânea s/n, 24210-340 Niterói RJ, Brazil \\ 2 Theoretical Physics, Department of Physics, Oxford University, 1 Keble Road, Oxford OX1 3NP
}

(October 29, 2018)

\begin{abstract}
Monolayer cluster growth in far-from-equilibrium systems is investigated by applying simulation and analytic techniques to minimal hard core particle (exclusion) models. The first model (I), for post-deposition coarsening dynamics, contains mechanisms of diffusion, attachment, and slow activated detachment (at rate $\epsilon \ll 1$ ) of particles on a line. Simulation shows three successive regimes of cluster growth: fast attachment of isolated particles; detachment allowing further $(\epsilon t)^{1 / 3}$ coarsening of average cluster size; and $t^{-1 / 2}$ approach to a saturation size going like $\epsilon^{-1 / 2}$. Model II generalizes the first one in having an additional mechanism of particle deposition into cluster gaps, suppressed for the smallest gaps. This model exhibits early rapid filling, leading to slowing deposition due to the increasing scarcity of deposition sites, and then continued power law $\left((\epsilon t)^{1 / 2}\right)$ cluster size coarsening through the redistribution allowed by slow detachment. The basic $(\epsilon t)^{1 / 3}$ domain growth laws and $\epsilon^{-1 / 2}$ saturation in model I are explained by a simple scaling picture involving the time for a particle to detach and diffuse to the next cluster. A second, fuller approach is presented which employs a mapping of cluster configurations to a column picture and an approximate factorization of the cluster configuration probability within the resulting master equation. This allows, through the steady state solution of the corresponding equation for a cluster probability generating function, quantitative results for the saturation of model I in excellent agreement with the simulation results. For model II, it provides a one-variable scaling function solution for the coarsening probability distribution, and in particular quantitative agreement with the cluster length scaling and its amplitude.
\end{abstract}

PACS numbers: 05.40.-a, 05.50.+q, 68.43.Jk

\section{INTRODUCTION}

This paper is concerned with domain growth in far from equilibrium systems. This is a subject of increasing interest both for the wide range of behaviors and for the large number of applications, which range from phase separation in mixtures to island formation and coarsening during deposition of a thin film or submonolayer ${ }^{1,2}$, among other systems.

Our aim is to discuss a series of one-dimensional exclusion models with particle diffusion, reversible or irreversible attachment to clusters and deposition mechanisms that represent, for example, volume reduction effects after cluster coalescence. Diffusion processes tend to bring these systems to equilibrium steady states, but pressure and other external influences may drive the system to new steady states. Though not usually related to a specific real problem, these one-dimensional models may reveal interesting features that help to understand more complex and realistic surface and bulk systems ${ }^{3,4}$, with the advantage of being more tractable both analytically and numerically. We will discuss a series of plausible physical situations in systems with diffusion and mechanisms that drive them out of equilibrium, in order to understand the details of domain growth and convergence to steady states, if it occurs.

In the first model, hereafter called model I, a fixed frac- tion $\rho$ of a one-dimensional lattice is randomly filled with hard core particles. The diffusion rates are $r=1$ when they are free, i. e. when they have two empty nearestneighbor sites, and $r=\epsilon \sim e^{-E / T}$ (where $E$ is the related energy barrier) when they have one occupied nearest neighbor site (Fig. 1a). For $\epsilon \ll 1(T \rightarrow 0)$, the average aggregates' length grows as $t^{1 / 3}$ in a long time range, and eventually approaches saturation at $\sim \epsilon^{-1 / 2}$ with a slow $t^{-1 / 2}$ decay (Sec. II). In the limit $\epsilon \rightarrow 0$, this model is equivalent to the Ising model with Kawasaki dynamics previously studied by Cornell et $\mathrm{al}^{5}$, who focused on its zero temperature features. However, the dynamic rules are mainly motivated by the Clarke-Vvedensky model for thin films or submonolayer growth ${ }^{6}$, excluding the deposition processes. In the simplest versions of that model, an isolated adatom has to overcome an energy barrier $E_{s}$ to diffuse, while when it is attached to $n$ nearest neighbors the energy barrier increases to $E_{s}+n E_{b}$, where $E_{b}$ is a bonding energy. This model and related ones were already intensively studied in two dimensions during the deposition process ${ }^{6-8}$, but a few works have considered the post-deposition coarsening dynamics ${ }^{9}$.

Subsequently we will generalize the previous hard core dynamical system by introducing deposition of particles (see e. g. Refs. ${ }^{11}$ and ${ }^{12}$ ), but allowing deposition only at (empty) sites with at least one empty nearest neighbor (Fig. 1b). In this model (referred to as model II), the 
domain coalescence, which generates larger vacancies between aggregates, is followed by a density increase. The exclusion of deposition at single holes between clusters represent the geometrical frustration of real systems. In Sec. III, we will show that this model exhibits a $t^{1 / 2}$ domain growth. This is among other results we have obtained by simulation studies, which are presented for both models I and II in Secs. II and III, respectively.

(a)
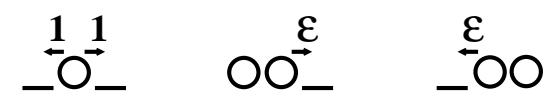

(b)
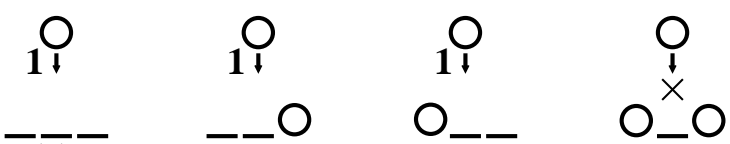

FIG. 1. (a) Diffusion and detachment processes of model I, with the corresponding rates. (b) Added deposition processes of model II, with the corresponding rates. The deposition at sites with two occupied neighbors (last process) is forbidden.

Our models share some aspects with diffusion limited coalescence models ${ }^{15-17}$ and with fragmentationaggregation models ${ }^{13,14}$. They are similarly described in terms of cluster or interval probabilities, and like the fragmentation models they are amenable to analytic investigation based on an independent cluster approximation (the independent interval approximation to the joint cluster length probability occurring in the master equation). We use this approach to explain properties of models I and II, including distributions of cluster size (Secs. II and III). Further, a simple scaling picture can be developed in order to describe the basic domain growth laws; we use this at the beginning of Sec. II.

\section{MODEL I: DIFFUSION, DETACHMENT AND REATTACHMENT OF PARTICLES}

\section{A. Processes}

The model studied in this section has the particle hopping processes depicted in Fig. 1a. Isolated particles hop symmetrically on a chain at unit rate ("diffusion"), while a single particle with a left hand / right hand neighbor can hop to an empty right / left neighbor with rate $\epsilon$ (detachment). So clusters evolve by detachment and reattachment of particles. The model is of exclusion type: no site can accommodate more than one particle.

This model is clearly particle-conserving, so density $\rho$ is fixed. The case $\epsilon \ll 1$ is of particular interest since, as reported in the simulation studies below and explained in the following subsection, very large clusters emerge.

\section{B. Simulations}

We simulated model I in one-dimensional lattices of length $L=8000$. This length is sufficiently large to ensure that finite size effects are negligible, as shown by comparisons of some results with data from lattices with $L=16000$ (particularly for the smallest values of $\epsilon$ this comparison is essential).

Initially, the lattice is randomly filled with a density of particles $\rho$. We simulated three values of the density, $\rho=0.1, \rho=0.5$ and $\rho=0.9$, which are representative of the range of intermediate densities, i. e. densities not too small $(\rho \approx 0)$ nor too large $(\rho \approx 1)$. For $\rho=0.1$ and $\rho=0.9$, we considered several values of the diffusion rate $\epsilon$ ranging from $\epsilon=10^{-1}$ to $\epsilon=10^{-3}$, and for $\rho=0.5$ we performed simulations until $\epsilon=10^{-5}$.

The sequence of characteristic behaviors of model I, as shown by simulation results, are: $(i)$ early fast attachment of isolated particles to each other to form clusters; (ii) an intermediate regime in which detachment sets in, allowing further coarsening; (iii) finally, there is a diffusive approach to a saturated state where the clusters have a large steady mean size that depends on $\epsilon$.

The three regimes are well separated at small $\epsilon$. This is illustrated in the plot of $\log _{10} d$ versus time $\log _{10} t$, shown in Fig. 2, where $d$ is the mean size of clusters of two or more particles; $d$ is given in terms of the probability $P_{t}(m)$ that an arbitrarily chosen cluster has size (or mass) $m$ at time $t$ by

$$
d=\frac{\sum_{m=2}^{\infty} m P_{t}(m)}{\sum_{m=2}^{\infty} P_{t}(m)} .
$$

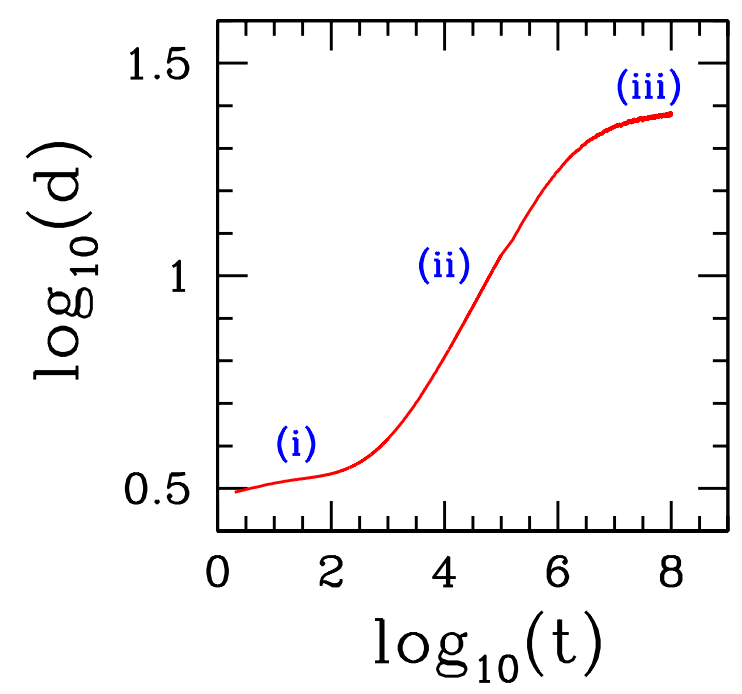

FIG. 2. Typical time evolution of the average cluster length $d$ in model I, with three different regimes. Data in the plot were obtained for $\rho=0.5$ and $\epsilon=10^{-4}$. 
The early time dependence of $d$ in region $(i)$ (at small $\epsilon)$ starts with a characteristic increase with rate proportional to a high power of $\epsilon$, and then crosses over to a form allowing data collapse in terms of the reduced time variable $\epsilon t$, as shown in Fig. 3 .

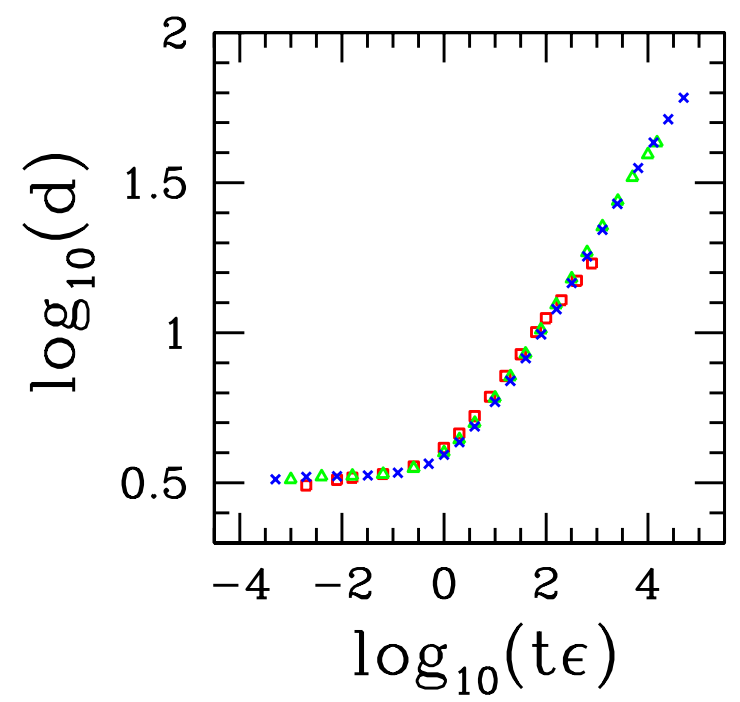

FIG. 3. Average cluster length as a function of the scaling variable $\log _{10}(\epsilon t)$, for model I with $\rho=0.5$ and $\epsilon=10^{-3}$ (squares), $\epsilon=10^{-4}$ (triangles) and $\epsilon=5 \times 10^{-5}$ (crosses).

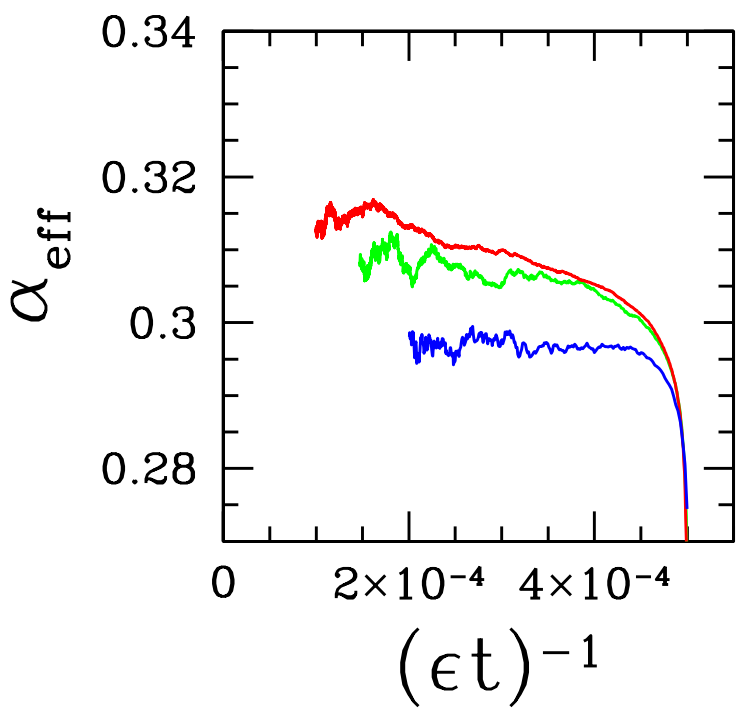

FIG. 4. Effective exponents $\alpha$, defined as local slopes of the $\log d \times \log t$ plots, for model I with $\rho=0.5$ and $\epsilon=5 \times 10^{-5}$ (below, blue), $\epsilon=2 \times 10^{-5}$ (medium, green) and $\epsilon=10^{-5}$ (up, red).
In region $(i i), d$ increases as

$$
d \sim t^{\alpha}
$$

The apparent exponent $\alpha_{e f f}$, defined as the local slope of the $\log d \times \log t$ plot, was calculated numerically. $\alpha_{e f f}$ is shown in Fig. 4 as a function of $(\epsilon t)^{-1}$ for three different values of $\epsilon$. It appears to approach the value $\alpha=1 / 3$ in the limit of small $\epsilon$ and correspondingly large $t$, which is consistent with the prediction of a simple scaling description (next subsection).

Fig. 5 shows the diffusive approach of the mean cluster size to its saturation value $d_{\infty}$. This approach is well described by $d=d_{\infty}-\frac{C}{t^{1 / 2}}$, for $t \rightarrow \infty$, with $C$ constant. The dependence on $\epsilon$ of the saturation value $d_{\infty}$ is illustrated in Fig. 6 for $\rho=0.5$. The least squares fit in Fig. 6 gives

$$
d_{\infty} \approx 0.72 \epsilon^{-1 / 2}+1.93
$$

in which the dominant (proportional to $\epsilon^{-1 / 2}$ ) and the sub-dominant (additive constant) terms were estimated. Like Eq. (2) with $\alpha=1 / 3$, this result follows from the analytic work in Sec. II C.

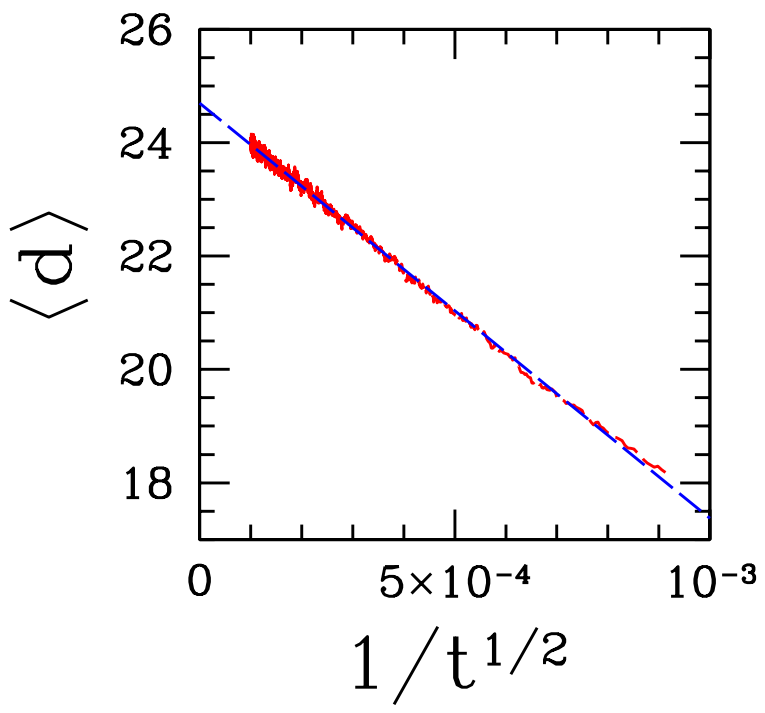

FIG. 5. Average cluster size at long times $t$ as a function of $1 / t^{1 / 2}$, for $\rho=0.5$ and $\epsilon=10^{-3}$ in model I. The dashed line is a least squares fit of the data for $10^{6} \leq t \leq 10^{8}$. 


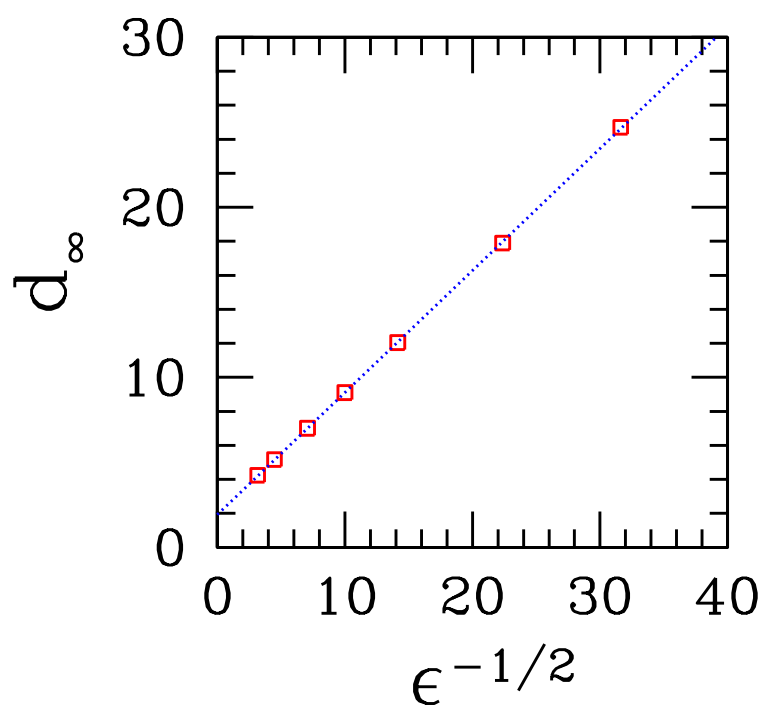

FIG. 6. Saturation value of the average cluster length as a function of $\epsilon^{-1 / 2}$ in model I. The dotted line is a least squares fit of the data.

\section{Theory}

The characteristic results just described have been interpreted by simple heuristic scaling arguments and by detailed analytic studies starting from the master equation and employing an independent interval approximation. This second approach is capable of yielding cluster length distributions and their evolution.

To begin with, we focus on the asymptotic cluster size at small $\epsilon$. This asymptotics occurs in the regime where lone particles are rare, and those that are present are in the process of reattaching themselves to the clusters they came from or to a neighboring one. The second case provides the sharing which sets the mean cluster size $d$. At densities of order $\rho \sim 1 / 2$, the cluster size is roughly of the order of cluster separation (see Fig. 7a). Thus the equilibrium of detachment time $(1 / \epsilon)$ and time of diffusion to a neighboring cluster $\left(\sim d^{2}\right)$ gives the observed saturation result

$$
d \sim \epsilon^{-1 / 2}
$$

This argument can be generalized rather obviously to explain the $t^{-1 / 2}$ approach to saturation.

A more interesting application is the explanation of the early cluster size growth law (Eq. 2). Here, unlike the saturation just described, the cluster separations are such that the detached particle is likely to return and reattach many times before it eventually diffuses to the next cluster (Figs. 7a and 7b). Its likelihood of returning to the origin means that the detachment rate $\epsilon$ needs to be replaced by an effective rate $\tilde{\epsilon}=\epsilon P_{m i g}$, where $P_{m i g}$ is the (migration) probability that a freely diffusing particle does not return to the detachment site before diffusing the distance $\sim d$ to the next cluster. In other words (see Fig. $7 \mathrm{~b}$ ), this is the probability that a free particle at position $y=1$ does not return to the origin $(y=0)$ before a time of order $d^{2}$, which is the typical time for diffusion along a distance $d$. Considering that

$$
Q(y, t)=\frac{y}{(4 \pi D)^{1 / 2}} t^{-3 / 2} \exp \left(-\frac{y^{2}}{4 D t}\right)
$$

is the probability that the first passage of a random walker at point $y$ occurs at time $t^{18}$ ( $D$ is the diffusion coefficient), $P_{m i g}$ is given by

$$
P_{m i g} \sim \int_{d^{2}}^{\infty} Q(1, t) d t \sim d^{-1} .
$$
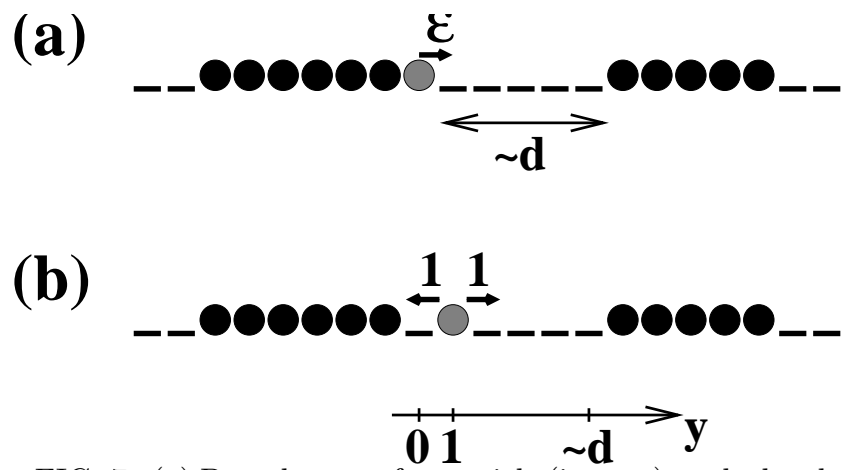

FIG. 7. (a) Detachment of a particle (in grey) at the border of a cluster, with rate $\epsilon$. In model I, the mean cluster length is $d$ and the typical cluster separation is, asymptotically, also of order $d$ for densities not too small nor too large. (b) A free particle (in grey) immediately after its detachment from the right cluster.

In terms of the effective rate $\tilde{\epsilon}$, the required time for a particle to transfer to the next cluster is of order $1 / \tilde{\epsilon}$. Thus, the time required for doubling the size $d$ of a cluster by successive gain and loss of particles is $d^{2} / \tilde{\epsilon} \sim d^{3} / \epsilon$. So the cluster growth proceeds according to

$$
\frac{d}{d t} d \sim \frac{d}{\left(d^{3} / \epsilon\right)}
$$

and hence

$$
d \sim(\epsilon t)^{1 / 3}
$$

This explains the behavior seen in the simulations (Figs. 3 and 4). The situation is analogous to domain scaling in Ising chains where, with Kawasaki dynamics ${ }^{5}$, spins split off from domain edges and migrate across to increase the domain size by one lattice unit.

We turn next to the more powerful analysis starting from a version of the master equation, which can provide 
a full description of the process. This is more easily set up by reformulating the process using a column picture, in which a column of height $m$ represents a cluster of size $m$, and then the original detachment and diffusion processes correspond to those shown in Fig. 8. Since one cluster has two edges but corresponds to a single column, the one-particle detachment rate in the column picture is

$$
\gamma=2 \epsilon
$$

\section{(a)}
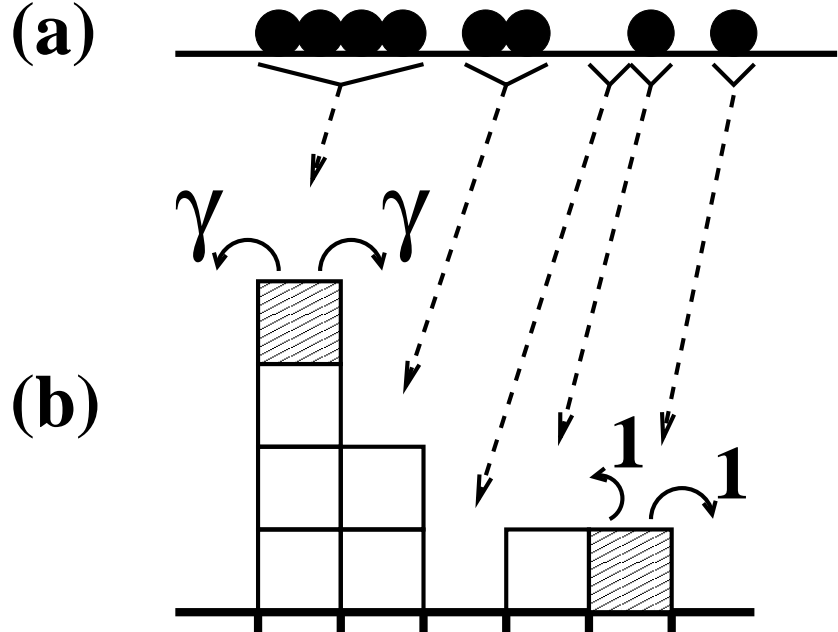

FIG. 8. (a) Example of particle-hole configuration on a line and the map (dashed arrows) into a column problem. (b) The processes of particle detachment from clusters, with rate $\gamma$, and of free particles diffusion, with rate 1 , in the corresponding column picture.

We denote by $P_{t}(m)$ the probability that a randomly chosen cluster (equivalently, column) has size $m$ at time $t$. Then the gain/loss from in/out processes provides the following master equation, in an independent interval approximation in which joint probabilities are factorized:

$$
\begin{aligned}
& P_{t+1}(m)-P_{t}(m)=\mathcal{A}_{m} \equiv \\
& P_{t}(m-1) \theta(m-1)\left[\gamma \sum_{m^{\prime} \geq 2} P_{t}\left(m^{\prime}\right)+1 \times P_{t}\left(m^{\prime}=1\right)\right]+ \\
& P_{t}(m+1)\left[\gamma \theta(m+1-2)+1 \times \delta_{m, 0}\right]- \\
& P_{t}(m)\left[\gamma \theta(m-2)+1 \times \delta_{m, 1}+\right. \\
& \left.\gamma \sum_{m^{\prime} \geq 2} P_{t}\left(m^{\prime}\right)+1 \times P_{t}\left(m^{\prime}=1\right)\right]
\end{aligned}
$$

The corresponding equation for the generating function

$$
G_{t}(s) \equiv \sum_{m=0} P_{t}(m) s^{m}
$$

is

$$
\begin{aligned}
G_{t+1}(s)= & G_{t}(s)\left[1+s a(t)+\frac{\gamma}{s}-\gamma-a(t)\right] \\
& +s(\gamma-1) P_{t}(1)+ \\
& {\left[\gamma P_{t}(0)+(1-\gamma) P_{t}(1)\right]-\frac{\gamma}{s} P_{t}(0), }
\end{aligned}
$$

where $a(t)=\gamma\left[1-P_{t}(0)\right]+(1-\gamma) P_{t}(1)$. It is easy to check probability and mass conservation using $G_{t}(0)$ and $G_{t}^{\prime}(0)$.

The steady state distribution $P(m)$ and generating function $G(s)$ resulting from Eq. (12) are given by

$$
\begin{gathered}
G(s)=(\gamma-s a)^{-1}[\gamma P(0)-s(1-\gamma) P(1)] \\
P(m)=\left(\frac{A}{\gamma}\right)^{m-1} P(1), m>1,
\end{gathered}
$$

and

$$
P(1)=A P(0)
$$

with $A=\gamma[1-P(0)][1-(1-\gamma) P(0)]^{-1}$. So the steady state cluster size distribution is exponential. The mean size of multi-particle clusters (Eq. 1) and the mean mass $\langle m\rangle \equiv \sum_{m=0}^{\infty} m P(m)$ are then obtainable in terms of $P(0)$, as is the density $\rho$. So, in particular the mean cluster length $d \equiv\langle m\rangle$ can be found in terms of $\rho$. The result simplifies at small $\gamma($ small $\epsilon)$ to

$$
\begin{aligned}
d= & \gamma^{-1 / 2}\left[\frac{\rho}{(1-\rho)}\right]^{1 / 2}+\frac{(\rho /(1-\rho)+3)}{2}= \\
& \epsilon^{-1 / 2}\left[\frac{\rho}{2(1-\rho)}\right]^{1 / 2}+\frac{(\rho /(1-\rho)+3)}{2},
\end{aligned}
$$

where the dominant and the first sub-dominant terms are shown. This form is consistent with the scaling result (4) and is in very good agreement with the simulation result (3), including the sub-dominant constant term: Eq. (16) gives $d=0.7071 \ldots \epsilon^{-1 / 2}+2$ for $\rho=0.5$ (see also Fig. $6)$. In the same limit, this is also, apart from a numerical factor, the characteristic size in the exponential cluster mass distribution.

\section{MODEL II: DIFFUSION AND DEPOSITION OF PARTICLES}

\section{A. Processes}

Model II is a generalization of model I, different only by having the deposition processes depicted in Fig. 1b, in addition to the diffusion and detachment processes of Fig. 1a. This makes the model non-particle-conserving, which leads to continued coarsening and other scaling properties and crossover. 


\section{B. Simulations}

The characteristic behavior of model II, as exhibited by simulation results, is as follows. For initial densities not too near $\rho=1$, there is: (i) an early regime of rapid filling, due to deposition, and cluster evolution due to both processes; (ii) an intermediate regime where deposition slows because of the scarcity of deposition sites due to the increased density - the exclusion constraint of course applies. The slow detachment process allows redistribution of particles, opening up new deposition sites and allowing the continually slowing coarsening (with no saturation as $\rho<1$ ).

Fig. 9 shows simulation results for the evolution of the mean cluster size $d$. That plot shows that $d(t)$ is well fitted by the form

$$
d(t)=B t^{1 / 2}\left(1+C t^{-1 / 2}+\ldots\right) .
$$

In Fig. 10 we show the ratio between the estimates of the amplitude $B$ and $\epsilon^{1 / 2}$ for several values of $\epsilon$. Those results give

$$
B(\epsilon) \sim b \epsilon^{1 / 2}
$$

with negligible corrections to scaling, where $b=0.252 \pm$ 0.002 . This result is in accord with theoretical analysis given in the next subsection, including the estimate of the amplitude $b$.

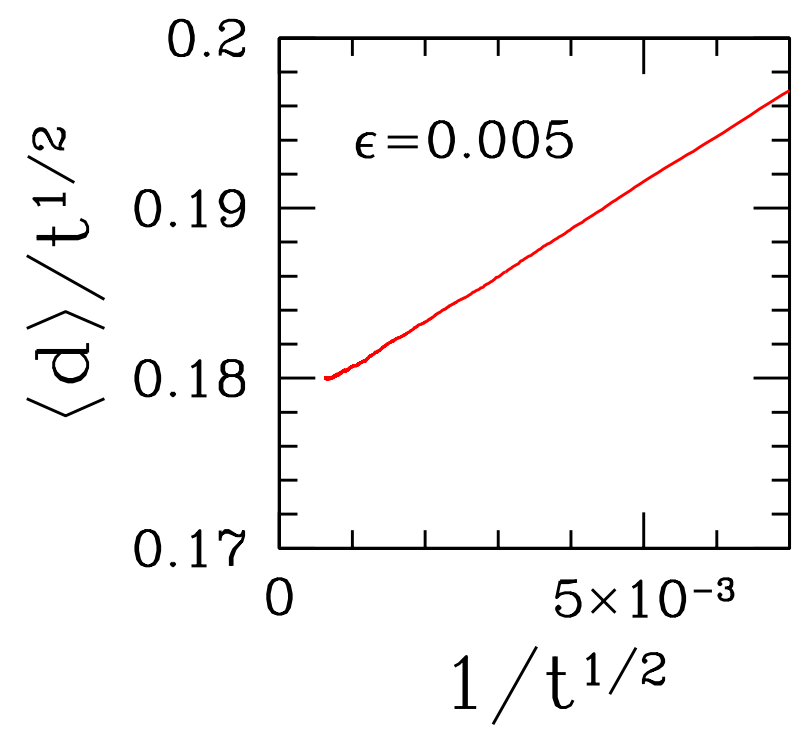

FIG. 9. Long time scaling of the mean cluster length $\langle d\rangle$ in model II.

The dependence of the evolving density on time $t$ and rate $\epsilon$ has also been studied. The simulation results shown in Fig. 11 imply that the density is a function of the scaling variable $(\epsilon t)^{1 / 2}$ and, at very long times, it converges to 1 as

$$
1-\rho \sim(\epsilon t)^{-1 / 2} .
$$

This form is also in agreement with the theoretical analysis below.

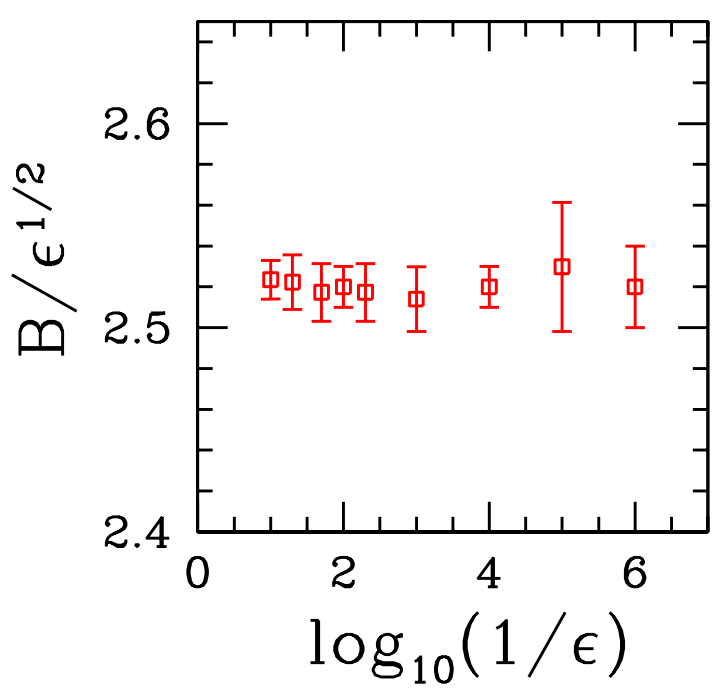

FIG. 10. Ratio between the estimated amplitude $B$ of the scaling of average cluster length (Eq. 17) and $\epsilon^{1 / 2}$, as a function of $\log _{10}(1 / \epsilon)$, in model II.

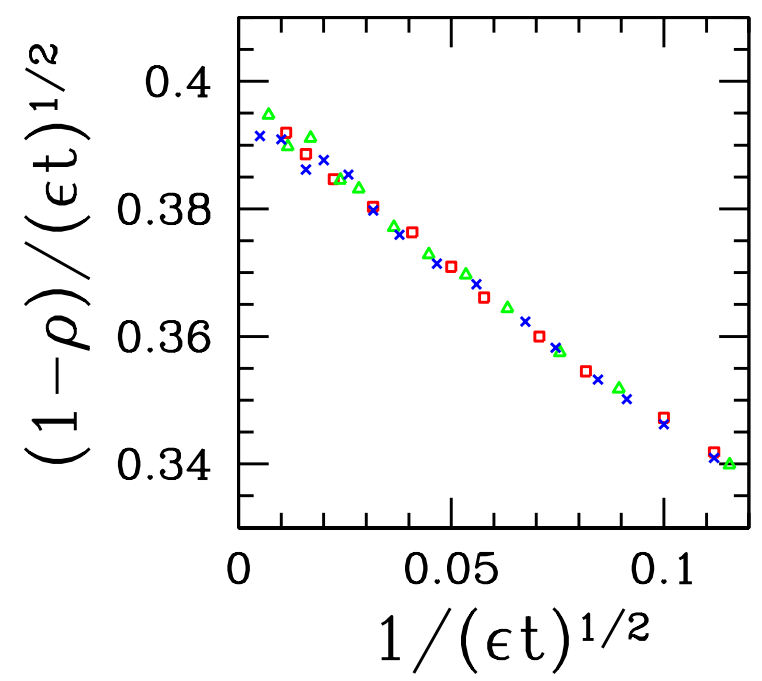

FIG. 11. Scaling plot of the particle density $\rho$ in model II, for $\epsilon=10^{-2}$ (squares), $\epsilon=5 \times 10^{-3}$ (triangles) and $\epsilon=10^{-3}$ (crosses). 


\section{Theory}

The characteristics presented in the foregoing subsection can be interpreted using an analytic investigation along the lines of the detailed discussion given in Sec. II C.

We have to include the effects of the extra deposition process, which leads to the decrease of the total number of clusters and of the number of holes between the clusters as time increases. On the other hand, the length $L$ of the line in which particles are deposited and diffuse is kept constant. Consequently, in order to adopt the column picture of Sec. II.3 (see Fig. 8), it is necessary to consider that the length $L_{0}$ of the corresponding column problem decreases in time (these lengths are related as $L_{0}=L-M$, where $M$ is the total mass or total number of particles, for periodic boundary conditions).

The evolution equation here is written for cluster numbers as

$$
N(m, t+1)-N(m, t)=L_{0}\left(\mathcal{A}_{m}+\mathcal{B}_{m}\right),
$$

where the diffusion contribution $\mathcal{A}_{m}$ is given in Eq. (10) and the deposition contribution is

$$
\begin{aligned}
& \mathcal{B}_{m}=P_{t}(0)\left[2 \theta(m-2) P_{t}(m-1)-\right. \\
& \left.2 \theta(m-1) P_{t}(m)+\delta_{m, 1} P_{t}(0)-2 \delta_{m, 0}\right] .
\end{aligned}
$$

The length of the lattice in which the column problem is defined varies due to deposition as

$$
\frac{L_{0}(t+1)-L_{0}(t)}{L_{0}(t)}=-P_{t}(0)\left[2-P_{t}(0)\right] .
$$

In these equations, the cluster probability is

$$
P_{t}(m)=\frac{N(m, t)}{L_{0}(t)} .
$$

They preserve conservation of probability, but mass is no longer conserved.

The resulting equation is similar to ones occurring in coalescence models ${ }^{15-17}$. From this we expect that a large time and small $\epsilon$ limit discussed subsequently is equivalent to the model in Ref. ${ }^{15}$. Our approach, which exploits the generating function method, becomes equivalent, in the scaling limit, though in a conjugate space, to continuum approximations used in the coalescence studies of Refs. ${ }^{15}$ and $^{17}$.

Now the generating function (Eq. 11) satisfies

$$
\begin{aligned}
& G_{t+1}(s)\left[1-P_{t}(0)\right]^{2}-G_{t}(s)= \\
& (s-1)\left[G_{t}(s)\left(a(t)-\frac{\gamma}{s}\right)+(\gamma-1) P_{t}(1)+\frac{\gamma}{s} P_{t}(0)\right]+ \\
& P_{t}(0)\left[2(s-1) G_{t}(s)-s P_{t}(0)+2\left(P_{t}(0)-1\right)\right]
\end{aligned}
$$

In the right hand site of Eq. (24), the first term corresponds to diffusion processes and the second one to deposition processes.
Because deposition slowly fills the system, we expect the configurations to coarsen and presumably to go into some scaling asymptotics where mass scales with some power of $t$, and $P_{t}(m)$ and $G_{t}(s)$ each become onevariable scaling functions. So we look for a long time scaling solution of the above equation.

At long times, the finite difference $G_{t+1}(s)-G_{t}(s)$ in Eq. (24) can be taken as a derivative. The scaling variable will be some combination of $t$ (large) and $u \equiv 1-s$ (small), the latter because large cluster sizes arise from structure in $G_{t}(s)$ at $s \approx 1$. The variable $u$ is actually conjugate to $m$ (see below). Coarsening will correspond to the scale of $m$ as $t^{z}$, with some power $z$, in which case the one-variable form will be

$$
G_{t}(s)=u^{\alpha} f\left(u t^{z}\right)
$$

with some function $f$. Normalization requires $\alpha=0$ and $f(0)=1$. In the scaling limit, the relationship of the generating function to the probability $P_{t}(m)$ requires the latter to be of the form

$$
P_{t}(m)=\frac{1}{t^{z}} g\left(\frac{m}{t^{z}}\right)
$$

with

$$
f(x)=\int_{0}^{\infty} g(y) e^{-x y} d y .
$$

It turns out that the consistent scaling solution has $g(0)=0$, so the $1 / t^{z}$ contribution to $P_{t}(0)$ vanishes, leaving a leading term of lower-than-scaling order,

$$
P_{t}(0)=\frac{c / 2}{t^{2 z}}
$$

where $c$ is a constant. Eq. (24) leads to the dynamical exponent

$$
z=1 / 2
$$

and to the following equation for the one-variable scaling function:

$$
x f^{\prime}(x)-2 c f(x)-2 \gamma x^{2} f(x)+2 c=0 .
$$

Even without solving Eq. (30) we can infer that

$$
P_{t}(0)=\frac{c / 2}{t}
$$

$$
\langle m\rangle_{t} \sim \gamma^{1 / 2} t^{1 / 2} \sim \epsilon^{1 / 2} t^{1 / 2}
$$

and

$$
1-\rho_{t} \sim \gamma^{-1 / 2} t^{-1 / 2} \sim \epsilon^{-1 / 2} t^{-1 / 2}
$$

These hold in the long time scaling limit we have introduced and agree with the observed simulation results in Eqs. (17), (18) and (19). 
Eq. (30) can be formally solved for the scaling function $f(x)$ by using the variable $\zeta=x^{2}$ and considering the function $f(x) x^{-2 c}$. The result is

$$
f(x)=\frac{c}{\gamma} \int_{0}^{\infty}\left(1+\frac{v}{\gamma}\right)^{-c-1} e^{-v x^{2}} d v .
$$

The large $x$ expansion of $f(x)$ and Eq. (27) provides the small $y$ expansion of $g(y)$ :

$$
g(y)=\frac{1}{\gamma^{1 / 2}} \mathcal{G}\left(\frac{y}{\gamma^{1 / 2}}\right)
$$

where

$$
\mathcal{G}(u)=\sum_{m=0}^{\infty} \frac{c(c+1) \ldots(c+m)}{(2 m+1) !}(-1)^{m} u^{2 m+1} .
$$

This confirms that $g(0)=0$. The cluster distribution has the following form, in terms of the odd function $\mathcal{G}$ :

$$
P_{t}(m)=\frac{1}{m^{*}(t)} \mathcal{G}\left(\frac{m}{m^{*}(t)}\right),
$$

where $m^{*}=(\gamma t)^{1 / 2} \sim(\epsilon t)^{1 / 2}$. It explains the scaling variable $(\epsilon t)^{1 / 2}$ used to collapse simulation data in Fig. 11.

The conditions that $\mathcal{G}$ must be non-negative and normalisable are satisfied with $c=1 / 2$ in Eq. (28), which leads to

$$
\mathcal{G}(u)=\frac{u}{2} e^{-(u / 2)^{2}}
$$

The mean cluster mass (cluster length in the original problem) is easily obtained as

$$
\langle m\rangle_{t} \approx \sqrt{\pi}(\gamma t)^{1 / 2}
$$

Considering relation (9), the amplitude of cluster length scaling is $B=\sqrt{2 \pi} \epsilon^{1 / 2} \approx 2.507 \epsilon^{1 / 2}$. It quantitatively agrees with the result obtained in simulations (Sec. III B).

\section{CONCLUSION}

We studied two one-dimensional exclusion models with particle diffusion, reversible or irreversible attachment to clusters and deposition mechanisms.

In model I, starting from a randomly filled lattice, only particle diffusion is allowed, with small detachment rates $\epsilon$ for particles at the edges of the clusters. Simulation results show an initial regime with formation of small clusters, a regime of cluster size growth as $d \sim t^{1 / 3}$ and a regime of cluster size saturation at $d \sim \epsilon^{-1 / 2}$. These results can be explained using heuristic scaling arguments. The analytical treatment of the master equation with an independent cluster approximation for joint probabilities distributions predicts a saturation cluster size in quantitative agreement with numerical data.

Model II generalizes model I in having also particle deposition: this is allowed only at empty sites with one or two empty nearest neighbors. Simulation results show continuous coarsening with a $t^{1 / 2}$ increase of the average cluster size and an increase of the density with $t^{-1 / 2}$ corrections. These scaling forms are justified by analytical investigations again using an independent cluster approximation, which provides good quantitative agreement with the simulations.

We expect that the models presented above and the combination of different methods to explain their scaling behaviors can be used to understand further nonequilibrium systems. Of particular interest would be the extension of theoretical methods (e. g. scaling approaches) to two-dimensional systems such as adatom islands on surfaces or the extension of the one-dimensional models to include other mechanisms that drive the systems to new non-equilibrium steady states or which lead to anomalous coarsening.

\section{ACKNOWLEDGMENTS}

FDAA Reis thanks the Department of Theoretical Physics at Oxford University, where part of this work was done, for the hospitality, and acknowledges support by CNPq and FINEP (brazilian agencies).

RB Stinchcombe acknowledges support from the EPSRC under the Oxford Condensed Matter Theory Grants, numbers GR/R83712/01 and GR/M04426.

${ }^{1}$ M. C. Bartelt and J. W. Evans, Phys. Rev. B 46, 12675 (1992).

${ }^{2}$ G. S. Bales and A. Zangwill, Phys. Rev. B 55, 1973 (1997).

${ }^{3}$ P. Sollich and M. R. Evans, Phys. Rev. Lett. 83, 3238 (1999).

${ }^{4}$ J. Jäckle and S. Eisinger, Z. Phys. B 84, 115 (1991).

${ }^{5}$ S. J. Cornell, K. Kaski, and R. B. Stinchcombe, Phys. Rev. B 44, 12263 (1991).

${ }^{6}$ S. Clarke and D. D. Vvedensky, J. Appl. Phys. bf 63, 2272 (1988).

${ }^{7}$ O. Barkema, O. Biham, M. Breeman, D. O. Boerma, and G. Vidali, Surf. Sci. 306, L569 (1994).

${ }^{8}$ C. Ratsch, A. Zangwill, P. Smilauer, and D. D. Vvedensky, Phys. Rev. Lett. 72, 3194 (1994).

${ }^{9}$ P.-M. Lam, D. Bayayoko, and X.-Y. Hu, Surf. Sci. 429, 161 (1999).

${ }^{10}$ I. Koponen, M. Rusanen, and J. Heinonen, Phys. Rev. E 58, 4037 (1998).

${ }^{11}$ J. W. Evans, Rev. Mod. Phys. 65, 1281 (1993).

${ }^{12} \mathrm{R}$. Stinchcombe, in Jamming and Rheology, eds. A. J. Liu 
and S. R. Nagel (Taylor and Francis, London and New York, 2001).

13 S. N. Majumdar, S. Krishnamurthy, and M. Barma, Phys. Rev. Lett. 81, 3691 (1998).

${ }^{14} \mathrm{~S}$. Krishnamurthy and R. B. Stinchcombe, to be published (2004).

${ }^{15}$ E. Abad, T. Masser, and D. ben-Avraham, J. Phys. A 35, 1483 (2002).

${ }^{16}$ D. ben-Avraham, M. A. Burschka, and C. R. Doering, J. Stat. Phys. 60, 695 (1990)

${ }^{17}$ D. Zhong and D. ben-Avraham, J. Phys. A 28, 33 (1995).

${ }^{18}$ E. W. Montroll and B. J. West, in Fluctuation phenomena, eds. E. W. Montroll and J. L. Lebowitz (North-Holland, Amsterdam, 1979). 